Article

\title{
Analysis of In-to-Out Wireless Body Area Network Systems: Towards QoS-Aware Health Internet of Things Applications
}

\author{
Yangzhe Liao $^{1, *}$, Mark S. Leeson ${ }^{1}$, Matthew D. Higgins ${ }^{2}$ and Chenyao Bai ${ }^{1}$ \\ 1 School of Engineering, University of Warwick, Coventry CV4 7AL, UK; \\ mark.leeson@warwick.ac.uk (M.S.L.); chenyao.bai@warwick.ac.uk (C.B.) \\ 2 WMG, University of Warwick, Coventry CV4 7AL, UK; m.higgins@warwick.ac.uk \\ * Correspondence: yangzhe.liao@warwick.ac.uk; Tel.: +44-759-647-6435 \\ Academic Editors: Enzo Pasquale Scilingo and Gaetano Valenza \\ Received: 6 April 2016; Accepted: 6 July 2016; Published: 13 July 2016
}

\begin{abstract}
In this paper, an analytical and accurate in-to-out (I2O) human body path loss (PL) model at $2.45 \mathrm{GHz}$ is derived based on a 3D heterogeneous human body model under safety constraints. The bit error rate (BER) performance for this channel using multiple efficient modulation schemes is investigated and the link budget is analyzed based on a predetermined satisfactory BER of $10^{-3}$. In addition, an incremental relay-based cooperative quality of service-aware (QoS-aware) routing protocol for the proposed I2O WBAN is presented and compared with an existing scheme. Linear programming QoS metric expressions are derived and employed to maximize the network lifetime, throughput, minimizing delay. Results show that binary phase-shift keying (BPSK) outperforms other modulation techniques for the proposed I2O WBAN systems, enabling the support of a $30 \mathrm{Mbps}$ data transmission rate up to $1.6 \mathrm{~m}$ and affording more reliable communication links when the transmitter power is increased. Moreover, the proposed incremental cooperative routing protocol outperforms the existing two-relay technique in terms of energy efficiency. Open issues and on-going research within the I2O WBAN area are presented and discussed as an inspiration towards developments in health IoT applications.
\end{abstract}

Keywords: health IoT; in-to-out body channel; WBAN; QoS-aware; BER; routing protocol

\section{Introduction}

The health Internet of Things (IoT) is one of the most promising approaches in improving the quality of human life. This is through healthcare monitoring and remote telemedicine support systems, which are able to deliver real-time data collection, transmission and visualization via the Internet [1,2]. In our previous work, we have presented a flexible quality of service (QoS) target-specific smart healthcare system [3]. This consisted of a smart gateway, a collection of sensor nodes and wireless communication links that can continuously acquire, process and transmit human vital signs to a remote medical server. Such a system makes remote patient health status monitoring by doctors and nurses a feasible proposition [1,3]. Moreover, the large volume of data collected makes it possible for researchers to develop new healthcare products and provide effective health education to people via the internet $[1,4]$. A wireless body area network (WBAN) is a networking technology that offers the prospect of the early detection of abnormal health conditions, real-time healthcare monitoring and remote telemedicine support systems. The Institute of Electrical and Electronic Engineers (IEEE) 802.15.6 Task Group has established the first international wireless communication standardisation of WBANs that optimises power consumption and provides safety guidance for medical and non-medical applications operating inside, on, or around the human body [4]. To date, the existing research work 
has mainly focused on on-body and off-body communication systems rather than in-body or I2O body communication systems $[1,4,5]$.

We previously proposed a biological implant WBAN communication channel [5], where the communication link was specific for the human cephalic region and covered a limited communication range. Thus in this paper, the proposed $\mathrm{I} 2 \mathrm{O}$ body WBAN systems enable transmission of real-time medical data from the in-body to the on-body area, which can enhance several practical health IoT scenarios [1,4]. Figure 1 shows the system in total, with the focus of this paper being the I2O tranmission link shown on the frontal thorax of the human outline.

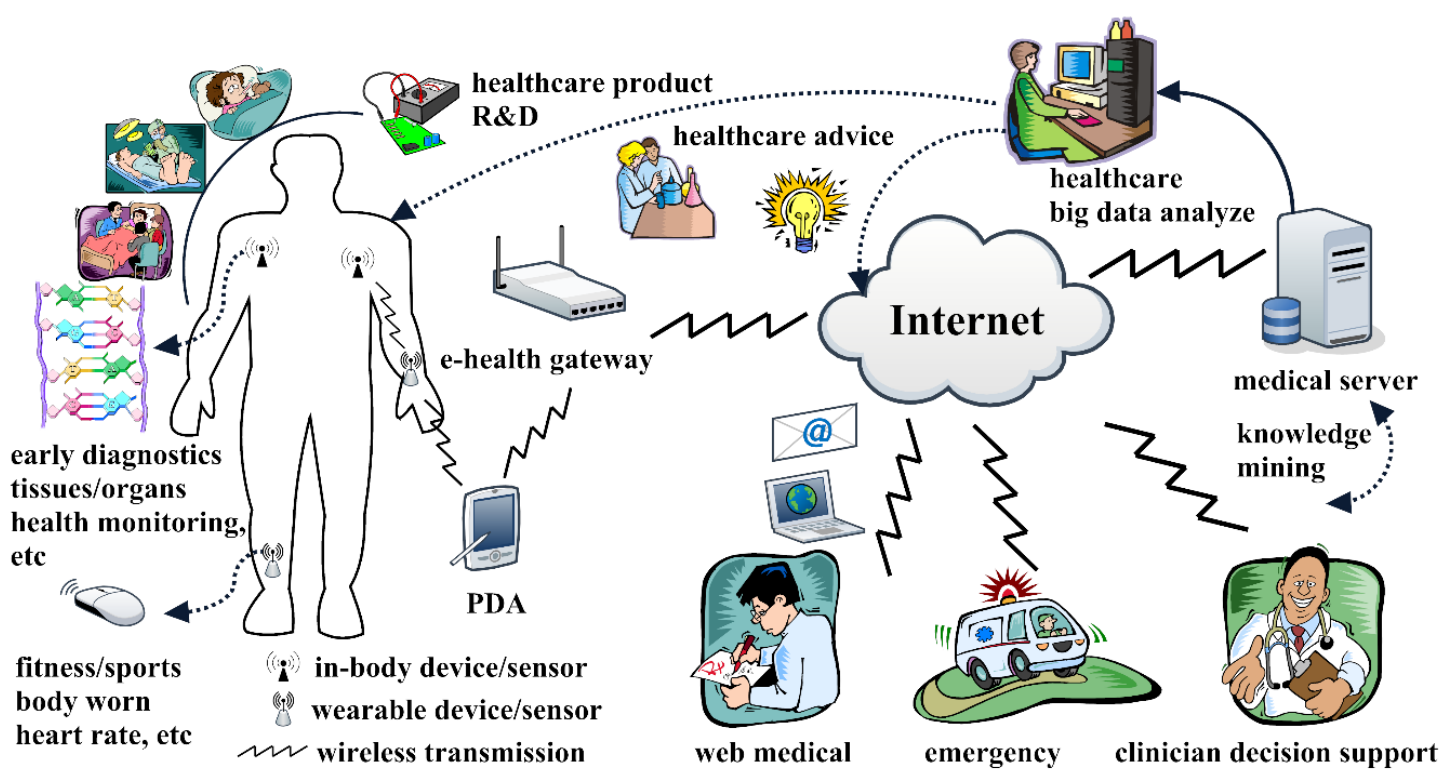

Figure 1. The proposed typical structure of the health IoT system.

WBANs differ from traditional wireless communication systems in terms of propagation medium, transmission power restrictions and human tissue/organ safety requirements [5]. Over 65\% of the human intra-body region is composed of water. Moreover, organ-tissue communications and drug transportation is via the blood [6]. This makes radio frequency (RF) signals remarkably attenuated when transmitting data through tissues/organs, even at relatively low frequencies such as the medical implant communication service (MICS) 402-405 MHz band proposed by the IEEE [5]. The 2.45 GHz industrial, scientific and medical (ISM) radio band is investigated in this article, as this brings advantages for WBAN systems. These are primarily that: (a) various compact small-sized antennas are available for implantation; (b) satisfactory performance is obtained with low energy consumption; (c) there is significant bandwidth to support high data transmission rates [4]. Increasing transmission power is one option to account for the energy attenuation but may result in damage to human tissues or organs due to heat absorption [4,7]. Thus, the transmission power must comply with the Federal Communications Commission (FCC) regulations and the specific absorption rate (SAR) must be lower than that laid down by IEEE and the International Commission on Non-Ionizing Radiation Protection (ICNIRP) $[8,9]$.

The concept of telemedicine has changed from 'medicine practiced at a distance' to 'personalised ubiquitous healthcare on the move' under the health IoT [1]. Concerning hospital or home healthcare monitoring scenarios, wireless implanted healthcare monitoring devices would significantly improve the comfort and mobility for patients when compared with wired connected medical devices. RF can cover longer operating distances and enable interactive implanted sensors and devices to communicate with on-body devices wirelessly [1,4,5]. Furthermore, due to the technical constraints of batteries, improving the energy efficiency of RF modules is a key factor in the $\mathrm{I} 2 \mathrm{O}$ body channel and one that can be realised by using small-sized implantable antennas [10-13]. 
Kurup et al. proposed the first in-body path loss (PL) model for homogeneous human muscle tissue at $2.45 \mathrm{GHz}$ in 2009 [14]. Later they reported an extended PL model, which includes the conductivity and permittivity of the human tissues in [15]. However, those PL models and other studies are antenna-specific and only applicable to the virtual family models provided by either of the SEMCAD X or FEKO software packages $[4,5,14,15]$. In this paper, the PL model is obtained by using software from Computer Simulation Technology (CST) [16] and the recently proposed 3D heterogeneous human body model developed by Kurup et al. [7]. The PL result is believed to be more accurate because the CST software takes the loss tangent parameters of human tissues and organs into account in the simulations and the deviation value is smaller than that seen in [11-15,17]. Furthermore, it is vital to choose suitable digital modulation techniques to overcome the strong power attenuation over the shadow fading channel caused by the intra-body environment [18]. The relationship between a large range of data rates from $250 \mathrm{kbps}$ to $30 \mathrm{Mbps}$ and the operating communication distance for safe transmission powers is discussed and investigated when using the selected modulation schemes.

Routing protocols have been reported to discover and analyze the most energy efficient route $[19,20]$. Relay based protocol solutions have also been studied that minimize the energy consumption of the in-body sensor nodes by reducing the length of the transmission distance [21]. A two relay based WBAN routing protocol has been investigated by Deepak and Babu [22] and the results show that the proposed routing scheme outperforms direct communication and single relay methods in terms of transmission reliability and energy efficiency. Cooperative communication techniques have gained significant attention as an effective strategy to improve energy efficiency and spatial diversity in wireless fading channels [21]. However, the incremental relaying routing protocol strategy has not been investigated in $\mathrm{I} 2 \mathrm{O}$ WBANs.

In this paper, an accurate statistical $\mathrm{I} 2 \mathrm{O}$ body path loss model that describes the signal propagation between the transmitter $(\mathrm{Tx})$ and receiver $(\mathrm{Rx})$ antennas is obtained by using CST electromagnetic solvers, based on a heterogeneous and innovative 3D virtual human body model at $2.45 \mathrm{GHz}$. The SAR is assessed to ensure adherence to the authorities' safety requirements. The BER performance for the I2O shadow fading channel employing the binary phase shift keying (BPSK), quadrature PSK (QPSK), 16 quadrature amplitude modulation (16QAM) and 16PSK modulation schemes is obtained. The threshold signal-to-noise ratio (SNR) of the four modulation methods is derived when using an acceptable BER performance of $10^{-3}$. Here, our proposed incremental relaying routing protocol is analyzed and compared with the existing two-relay WBAN routing scheme. A series of QoS metrics such as network lifetime, throughput, average energy consumption, residual energy and propagation delay are also investigated.

The rest of the paper is organized as follows: In Section 2, we briefly introduce a typical I2O WBAN system; details of our proposed I2O WBAN simulation setup, path loss, channel modeling and link budget are given in Section 3; comparison of the proposed incremental relaying protocol and the existing two-relay protocol, including the analysis of mathematical expressions of related QoS metrics, communication flow and QoS metrics performance is shown in Section 4; Section 5 analyzes numerous emerging I2O WBAN topics and health IoT requirements; finally, Section 6 presents the conclusions.

\section{System Model}

Health IoT communication systems cover short-range telemetry communication channels such as from in-body to on-body. The on-body device can also collect and transmit high data rates and real-time medical information to doctors and nurses via a gateway [1,3]. Figure $2 \mathrm{a}-\mathrm{c}$ illustrates the elements of the CST model, whilst the three primary components of the health IoT WBAN system are shown in Figure 2d with further details given below:

- Implant device/sensor: A biological compatible and miniaturized size implant device that is located inside the human body, either in the tissue/organ region (deep region) or under the skin (near surface) [10].

- On-body device/sensor: An on-body (or wearable) device that can be located on either surface or up to $20 \mathrm{~mm}$ away from skin [2]. 
- Gateway: Typically, this has no direct connection to an implanted device or sensor. A smartphone or other personal data device is needed to enable to the collection, processing and transmission of data to doctors and nurses via the internet [3].

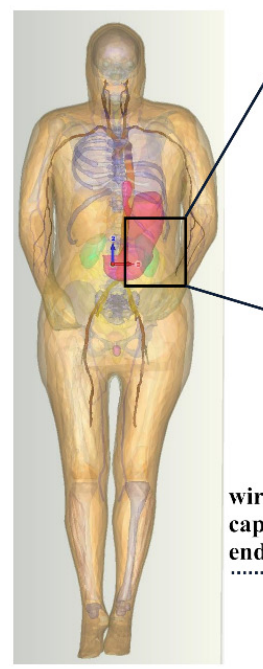

(a)

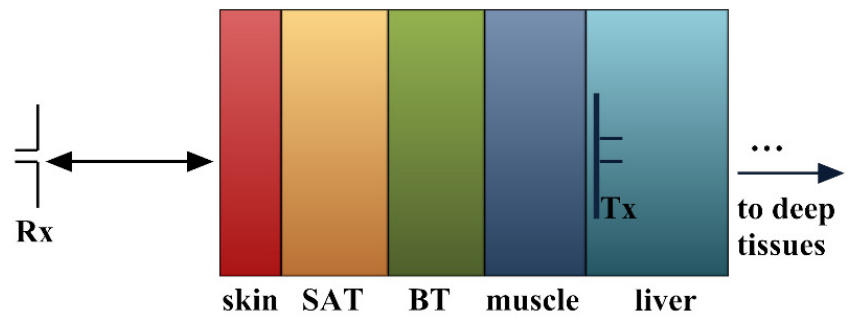

(c)

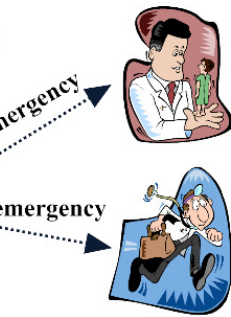

(d)

Figure 2. (a) The computational 3D human body model in CST; (b) the cross section of the human frontal thorax; (c) equivalent human frontal thorax model; (d) typical healthcare applications.

\section{Analysis of I2O WBAN Systems}

In this section, the proposed communication system is considered in likely operating scenarios, such as in hospitals or homes, where a patient with an in-body implant device/sensor transmits data to a wearable device placed at the maximum distance of the on-body region. A communication link is established for an $\mathrm{I} 2 \mathrm{O}$ body scenario between the Tx located in the liver tissue and the Rx placed $2 \mathrm{~cm}$ from the human body surface. The BER performance for the $\mathrm{I} 2 \mathrm{O}$ shadow fading channel employing BPSK, QPSK, 16PSK and 16QAM modulation schemes is obtained. The relationship between the data transmission rates, transmitting power and the achievable communication distance is discussed under the link budget analysis.

\subsection{Configuration and Human Safety Analysis}

Implantable biomedical antenna design in WBAN systems is affected by posture, body mass index, aging and so on. Detailed information about biocompatible in-body antenna design can be found in the review by Movassaghi et al. [4]. Here, we focus our attention mainly on I2O body communication systems analysis. An efficient and multi-layered heterogeneous human body model (Figure 3) proposed in [23] for WBANs, at the frequency of $2.45 \mathrm{GHz}$, is investigated in this paper. The configuration consists of a layer of air followed by layers equivalent to the frontal thorax of an adult, making it possible to divide the human body into numerous areas of a multi-layered model. We employ dipole antennas because they are well-studied antennas in free space and have a simple structure [5,15,17]. In agreement with [23], we place the Tx antenna of length $3.9 \mathrm{~cm}$ in the liver region and the Rx antenna is a free space, half wavelength dipole with a length of $6.12 \mathrm{~cm}$, located $2 \mathrm{~cm}$ from the human body surface. Both the Rx and Tx antennas are made of perfect electric conductors (PEC) and are directionally aligned, with a thickness of $2 \mathrm{~mm}$. The simulations use a current source and the simulation methods are the same for all the cases. The equivalent 3D human body model contains multiple layers that are built on dry skin, subcutaneous adipose tissue (SAT), breast tissue (BT), muscle tissue and liver; whose thicknesses are $2 \mathrm{~mm}, 5 \mathrm{~mm}, 1 \mathrm{~mm}, 10 \mathrm{~mm}$ and $10 \mathrm{~mm}$ with dielectric properties as shown in Table 1. Since it is difficult for both manufacturers and researchers to 
investigate their systems on an actual human body, the proposed human body model offers a viable alternative to investigate the performance of I2O body WBAN systems.

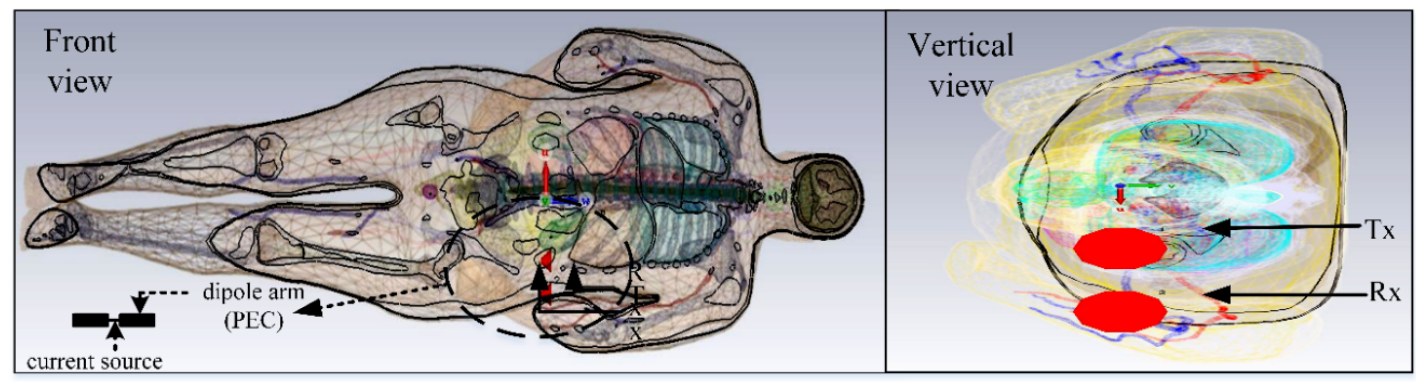

Figure 3. The 3D human body model and dipole antennas. (left) front view; (right) vertical view.

Table 1. Conductivity $\left(\varepsilon_{r}\right)$, Relative Permittivity $\sigma$ and Loss Tangent $\tan \delta$ [24].

\begin{tabular}{cccccc}
\hline Parameter & Skin & SAT & BT & Muscle & Liver \\
\hline$\varepsilon_{\mathrm{r}}$ & 38 & 10.8 & 5.15 & 52.7 & 43 \\
$\sigma[\mathrm{S} / \mathrm{m}]$ & 1.46 & 0.27 & 0.14 & 1.74 & 1.69 \\
$\tan \delta$ & 0.28262 & 0.14524 & 0.19535 & 0.24194 & 0.28751 \\
\hline
\end{tabular}

As reported in [5,7], signal propagation in the human body leads to high attenuation, which will result in heating of tissues and organs and to an increase in the temperature of the human body. Biological effects and health risks may occur by exposure to RF electromagnetic fields. The IEEE standard and the ICNIRP safety guidance specify that the averaged SAR over $10 \mathrm{~g}$ of tissue should be no more than $1.6 \mathrm{~W}$ per $\mathrm{kg}$ and $2 \mathrm{~W}$ per $\mathrm{kg}$, respectively [8,9]. With an input power of $1 \mathrm{~W}$ provided to the implanted sensor or device, the finite-difference time domain (FDTD) approach provided by CST was used in association with the 3D human model investigated to calculate the SAR [25]. Results demonstrate that the SAR of the human body model is far lower than both the regulations. The maximum $1 \mathrm{~g}$ and $10 \mathrm{~g}$ SAR values calculated tissue are given in Table 2 for the skin minimal distance (skin region) and maximum distance (liver region).

Table 2. Typical Locations and Values of $1 \mathrm{~g}$ and $10 \mathrm{~g}$ SAR for $\mathrm{I} 2 \mathrm{O}$ Body Model.

\begin{tabular}{ccc}
\hline Distance & Maximum SAR (1 g) & Maximum SAR (10 g) \\
\hline $5 \mathrm{~mm}$ & $36.8 \mathrm{~mW} \cdot \mathrm{kg}^{-1}$ & $17.4 \mathrm{~W} \cdot \mathrm{kg}^{-1}$ \\
$20 \mathrm{~mm}$ & $31.5 \mathrm{~W} \cdot \mathrm{kg}^{-1}$ & $19.3 \mathrm{~W} \cdot \mathrm{kg}^{-1}$ \\
\hline
\end{tabular}

\subsection{Path Loss Model}

The human body is a natural lossy environment, which therefore leads to high attenuation for signal transmission. The PL model of homogeneous human tissue/organ has been described in [14]. For the heterogeneous human body, the Tx antenna moves through the different layers (tissues) and the PL is obtained. When the antenna is placed in a specific layer, the surrounding layers differ from that containing the antenna, leading to deviations between the simulated and calculated PL values. A semi-empirical PL formula in $\mathrm{AB}$ between two implant devices can be expressed as $[5,14,15,25]$ :

$$
P L_{d B}(d)=P L_{d B}\left(d_{r e f}\right)+10 n \log _{10}\left(\frac{d}{d_{r e f}}\right)+S_{d B}, d \geqslant d_{r e f}
$$

where $P L_{d B}\left(d_{r e f}\right)$ is the PL value at the reference distance $d_{r e f}\left(5 \mathrm{~mm}\right.$ in this paper). $S_{d B}$ is the shadow fading parameter expressed in decibels $(\mathrm{dB})$, which follows a normal distribution $S \sim N\left(0, \sigma_{s}\right)$ where $\sigma_{S}$ is the standard deviation, since the logarithm of a lognormal distribution is normally distributed. 
Moreover, $\sigma_{s}$ reflects the degree of the shadow fading strength [25]. The probability density function of the shadow fading effect $S_{d B}$ can be expressed as:

$$
P_{S_{d B}}=\frac{1}{\sigma \sqrt{2 \pi}} \exp \left(-\frac{S_{d B}^{2}}{2 \sigma_{\log }^{2}}\right)
$$

The variable $d$ is the separation distance between the Tx and Rx; $n$ is the path loss exponent which depends on the propagation media. MATLAB ${ }^{\circledR}$ least square fit computation has been implemented to yield a fitted PL as seen in Figure 4. The simulation results are summarized in Table 3. Closer agreement than many proposed PL models $[15,16]$ is obtained between the derived PL model and the simulations using CST with an average deviation of $2.93 \mathrm{~dB}$.

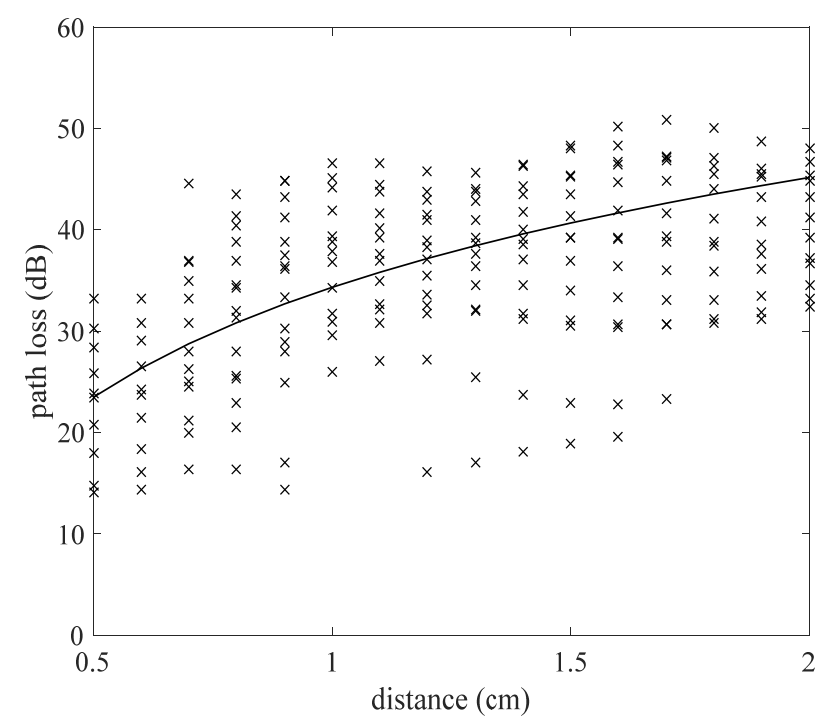

Figure 4. Path loss model versus distance between Tx and Rx antennas.

Table 3. Simulation Results of the Path Loss Model.

\begin{tabular}{ccc}
\hline Parameter & Value (Unit) & Description \\
\hline$n$ & 3.6 & PL exponent \\
$\sigma_{d B}$ & 2.93 & average deviation \\
$d_{r e f}$ & $0.5 \mathrm{~cm}$ & reference distance \\
$P L_{d B}(r e f)$ & $23.49 \mathrm{~dB}$ & PL at the reference distance \\
\hline
\end{tabular}

\subsection{I2O Channel Model}

Energy efficient modulation schemes are beneficial in reducing the hardware structure, lowering the noise interference and prolonging the communication network lifetime, due to the shortage of implanted device battery capacity. Moreover, low error probabilities are required in the modulation methods to ensure the reliability of the communication transmission. In [18], M-ary PSK (M-PSK) is reported to achieve significant energy saving and high system outage probability. In addition, 16QAM is the most spectral efficient modulation technique, which can meet higher data rates than QPSK and 8PSK when operating under the same bandwidth $[10,26,27]$. An I2O communication channel shares the property with all fading RF channels that the instantaneous SNR, $\gamma$, at the receiver side, can be regarded as a random variable due to the fading effect. Since this means that the bit errors in demodulation are therefore not fixed since the SNR varies randomly, we investigate the expected bit error rate (BER) for channel performance evaluation, where the expectation is with respect to the probability distribution of the SNR. 
The average BER of the $\mathrm{I} 2 \mathrm{O}$ shadow fading communication channel can be evaluated by the following integral [5,28]:

$$
P_{e}(\bar{\gamma})=\int_{0}^{\infty} P_{b, A W G N}(\gamma) P_{0}(\gamma) d \gamma
$$

where $P_{b, A W G N}(\gamma)$ represents the BER performance of the additive white Gaussian noise (AWGN) channel for an SNR, $\gamma$, and assuming a mean SNR, $\bar{\gamma}$. The function $P_{0}(\gamma)$ denotes the probability density function of the $\gamma$, which is lognormal distributed with the same standard deviation $\sigma_{s}$ as the BER performance for the $\mathrm{I} 2 \mathrm{O}$ shadow fading channel when employing the four selected modulation techniques. The received power can be obtained simply from the transmitted power $P_{t}$ and PL via:

$$
P_{r}=\frac{P_{t}}{P L}
$$

The received energy per bit $E_{b}$ can be related to the data rate $R_{b}$ and the received power and it then can be expressed as:

$$
\frac{E_{b}}{N_{o}}=\frac{P_{r}}{N_{0} R_{b}}=\frac{P_{t}}{R_{b} N_{0} P L}
$$

where $N_{0}$ is the noise power. Expressing (6) in $\mathrm{dB}$ :

$$
\ln \left(\frac{E_{b}}{N_{o}}\right)=\ln \left(\frac{P_{t}}{R_{b} N_{0} P L}\right)=\ln \left(\frac{P_{t}}{R_{b} N_{0}}\right)-\ln P L
$$

The first term in the Expression (6) for $\ln \left(E_{b} / N_{o}\right)$ is fixed for a given data rate $R_{b}$ and transmitter power $P_{t}$. Thus, since the PL model presented earlier follows a lognormal distribution, $\ln \left(E_{b} / N_{o}\right)$ is normally distributed, i.e., $E_{b} / N_{0}$ follows a lognormal distribution:

$$
P_{0}(\gamma)=\frac{1}{\sqrt{2 \pi} \sigma \gamma} e^{-\frac{(\ln \gamma-\mu)^{2}}{2 \sigma^{2}}}
$$

where $\mu=\ln \bar{\gamma}-\frac{1}{2}\left(\frac{\ln 10}{10}\right)^{2} \sigma_{d B}^{2}$, and $\sigma=\frac{\ln 10}{10} \sigma_{d B}$.

Detailed information on the four selected modulation techniques can be found in $[5,18,26,27]$. We use coherent BPSK modulation as a concrete example, where [5]:

$$
P_{b, A W G N}(\gamma)=\frac{1}{2} \operatorname{erfc}(\sqrt{\gamma})
$$

where $\operatorname{erfc}($.$) is the complementary error function and so (3) becomes:$

$$
P_{e}\left(\bar{\gamma}_{m}\right)=\sum_{n=1}^{N} \frac{1}{2} \operatorname{erfc}(\sqrt{\gamma}) \frac{1}{\sqrt{2 \pi} \sigma \gamma_{n}} e^{-\frac{\left(\ln \gamma_{n}-\ln \bar{\gamma}_{m}+\frac{1}{2}\left(\frac{\ln 10}{10}\right)^{2} \sigma_{d B}^{2}\right)}{2 \sigma^{2}}}\left(\gamma_{n}-\gamma_{n-1}\right)
$$

The average BER performance of the $\mathrm{I} 2 \mathrm{O}$ fading channel under BPSK is then obtained by numerical evaluation of $(9)[5,28]$. The same method can be applied to other proposed modulation schemes. The average BER performance of the four selected modulation methods is shown in Figure 5. At the receiver, AWGN is the dominant noise source [5] so we consider only thermal noise with one-sided power spectral density (PSD) [5,29-31]:

$$
N_{0}=k\left[T_{I}+\left(N_{F}-1\right) T_{O}\right]
$$

where $k$ and $N_{F}$ are the Boltzmann constant and receiver noise factor, respectively; $T_{I}$ and $T_{O}$ are the noise temperatures at the receiver and transmitter. The mean temperature of liver tissue is around the $306 \mathrm{~K}$ and the ambient temperature of human skin is $310 \mathrm{~K}[24,32] . N_{F}$ is the noise figure and can be defined as:

$$
N_{F, d B}=10 \log _{10}\left(N_{F}\right)
$$


where $N_{F}=1+T_{I} / T_{O}$. The received SNR in $\mathrm{dB}$ can be expressed as:

$$
S N R_{d B}=P_{r, d B W}-10 \log _{10}\left(R_{b}\right)-N_{F, d B}
$$

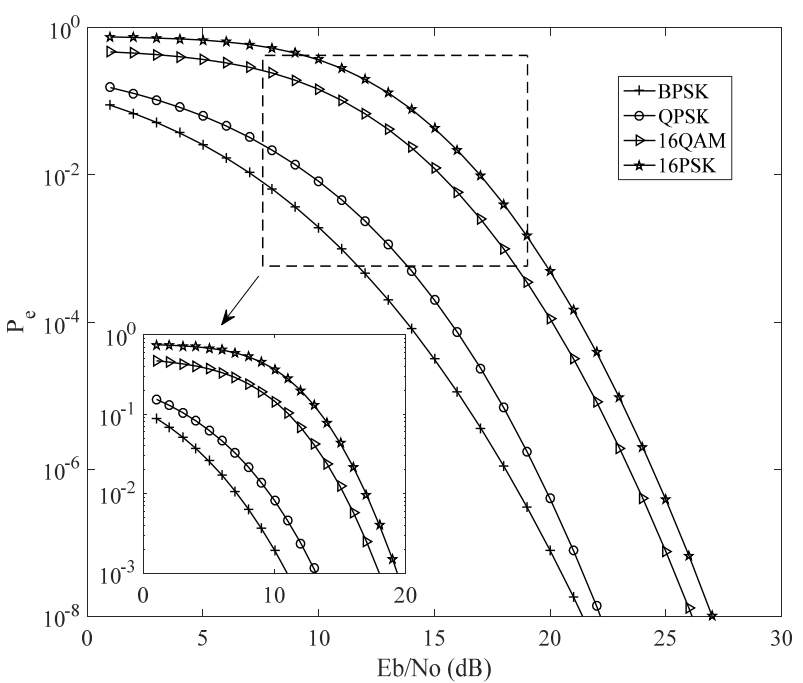

Figure 5. BER performance comparison between BPSK, QPSK, 16QAM and 16PSK

\subsection{Link Budget Analysis}

The $\mathrm{I} 2 \mathrm{O}$ human body environment is lossy producing and high attenuation, so it is important to analyze the link budget when designing wireless communication systems for several scenarios. Two significant elements are: (i) an energy consumption calculation for a required communication link quality; (ii) the effective communication distance estimation when employing a certain transmitting power.

Based on the European Research Council (ERC) regulations, the maximum input power is $25 \mu \mathrm{W}$ [19]. In this paper, typical transmitting power values of 1,10 and $25 \mu \mathrm{W}$ are selected for further research and discussions. Given the high QoS requirements of healthcare communication systems, similar to other I2O body communication systems, a predetermined BER threshold of $10^{-3}$ was selected to ensure the communication performance is acceptable [5,28-31]. According to Figure 5, the threshold $S N R_{t h r}$ values for BPSK, QPSK, 16QAM and 16PSK are approximately $11 \mathrm{~dB}, 13 \mathrm{~dB}$ and $15.5 \mathrm{~dB}$ and $18 \mathrm{~dB}$, respectively. The parameters used in the link budget simulations are summarized in Table 4 .

Table 4. Parameters for the Link Budget Simulations.

\begin{tabular}{cc}
\hline Simulation Parameter & Value \\
\hline Frequency band $(\mathrm{GHz})$ & 2.45 \\
\hline Tx output power $(\mu \mathrm{W})$ & $1,10,25$ \\
\hline Antenna gain $(\mathrm{dBi})$ & 0 \\
\hline Coding gain $(\mathrm{dB})$ & 0 \\
\hline Ambient temperature $(\mathrm{K})$ & 310 \\
\hline Liver tissue temperature $(\mathrm{K})$ & 306 \\
\hline Boltzmann constant $\left(\mathrm{JK}{ }^{-1}\right)$ & $1.38 \times 10^{-23}$ \\
\hline BER $($ predetermined $)$ & $10^{-3}$ \\
\hline SNR (threshold) $(\mathrm{dB})$ & $11(\mathrm{BPSK}), 13(\mathrm{QPSK})$ \\
- & $0.25,5,30$ \\
Selected data rate $(\mathrm{Mbps})$ & 2 \\
\hline Selected distance $(\mathrm{m})$ &
\end{tabular}

One valuable system parameter that can effectively evaluate the reliability of the communication system using the threshold BER performance is the system margin, $M_{s}$. A communication channel 
with a negative link margin has insufficient power to transmit data and thus, is essential to offer adequate link to ensure that the communication system is reliable [33]. $M_{S}$ is given by determining the SNR above the threshold level $\left(S N R_{t h r}\right)$ as:

$$
M_{S}=S N R_{d B}-S N R_{t h r}>0
$$

Figures 6-9 show the dependence of system margin versus communication link distance, with several levels of data rates and multiple modulation techniques utilizing transmitter powers of $1 \mu \mathrm{W}, 10 \mu \mathrm{W}$ and $25 \mu \mathrm{W}$. For health IoT applications, such as hospital real-time healthcare monitoring services, where the receiving sensor or device is normally placed 1 meter away from patients, all the above-mentioned methods could support satisfactory wireless data transmission $[1,2,23]$. Due to the constraints on the battery energy supply of the implantable device or sensor, trade-offs between transmitting power and communication channel quality should be taken into account $[11,12]$. Results show that, as one might expect, higher transmitting power can achieve longer communication distances for a certain data rate. The communication system can also cover longer distances by using lower transmission data rates when compared with higher data rates. For conditions when under the same data rates and transmitting powers, the BPSK modulation scheme can achieve more reliable transmission than the other investigated modulation schemes. Furthermore, we suggest a fade system margin of a few dBs that accounts for energy losses caused by the antenna orientations and body movements to ensure the reliability of the data transmission, when designing real-time healthcare wireless monitoring systems.

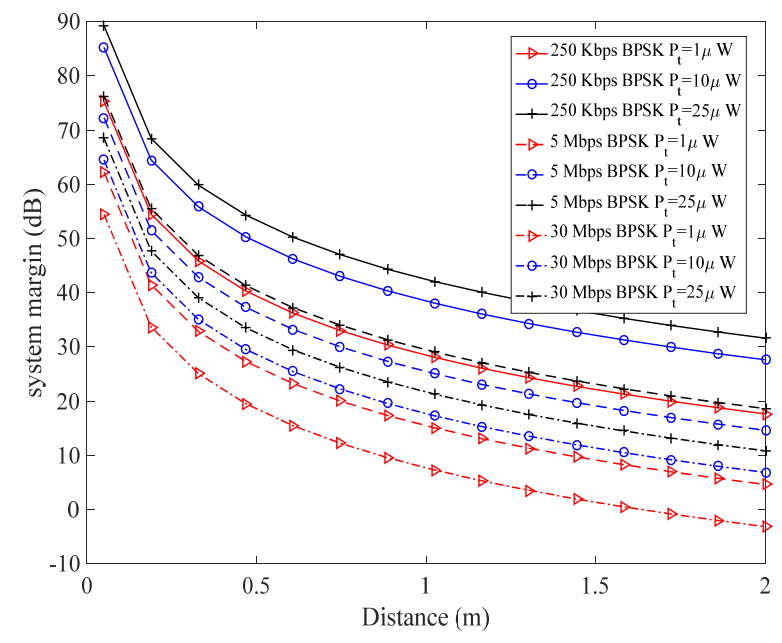

Figure 6. Link margin versus distance using BPSK at multiple levels of data rates and powers.

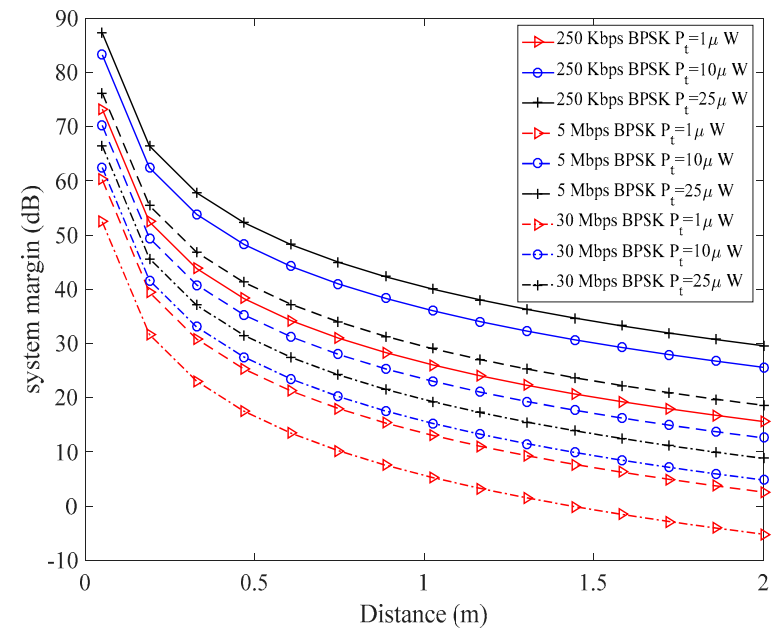

Figure 7. Link margin versus distance employing QPSK at multiple levels of data rates and powers. 


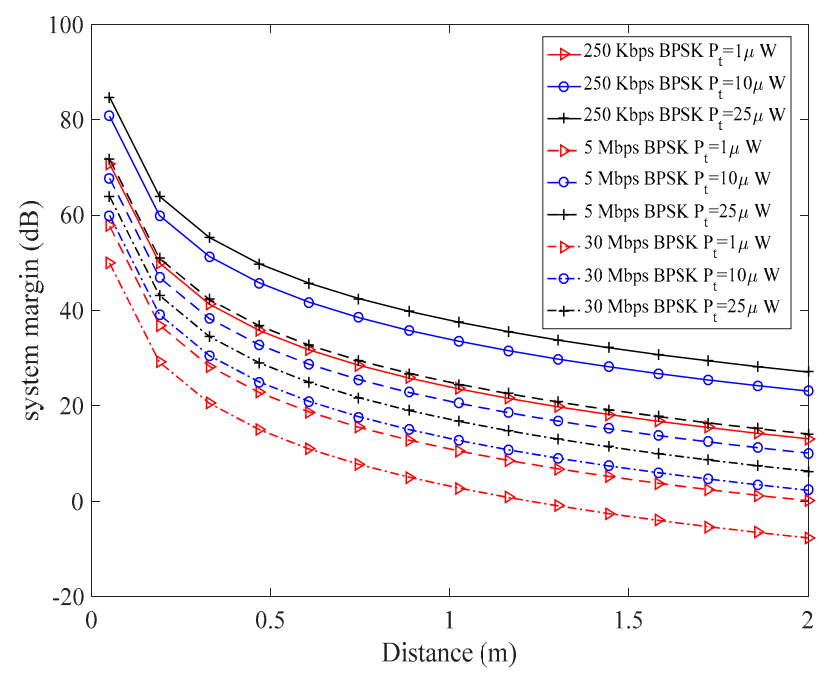

Figure 8. Link margin versus distance for 16PSK at multiple levels of data rates and powers.

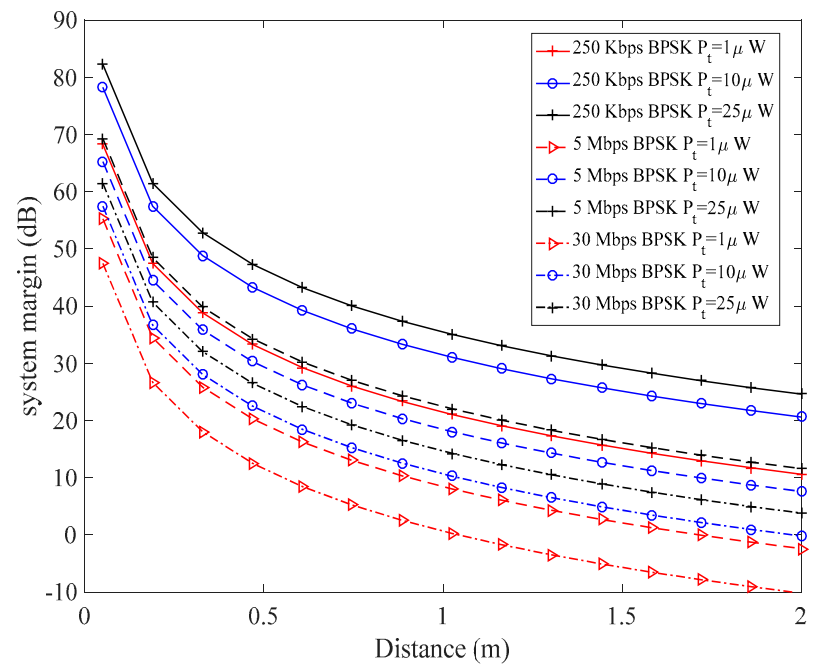

Figure 9. Link margin versus distance for 16QAM at multiple levels of data rates and powers.

\section{Relay Based QoS-Aware Routing Protocol for I2O WBAN}

\subsection{Motivation}

One of the major challenges in I2O WBANs is maximizing the WBAN lifetime [21]. To date, there are several routing protocols that have been reported in literature such as single or two-relay WBAN techniques [22]. However, those routing protocols are not very energy efficient and less likely to support long-term healthcare monitoring tasks. In [23], the authors stated that the energy consumption of implants is directly related to the transmission distance and therefore, energy efficient routing protocols are an effective approach in minimizing the overall length of communication paths. Moreover, by deploying an incremental relaying strategy, the complexity and energy consumption are transferred from the implant device to the on-body relay, which is a device that can be easily replaced and recharged, in contrast to the in-body sensor nodes [10,34]. QoS requirements in WBANs vary between applications [35]. A practical approach is to focus on data transmission models that are used in different applications and map the requirements of these onto a set of QoS metrics [35]. Figure 10 demonstrates the multiple relay-based routing protocols proposed in [21,22]; Figure 10a, single relay based scenario; Figure 10b-d, two-relay based selective routing techniques. 
In this section, mathematical formulas for important QoS metrics in terms of network lifetime, network throughput and end to end delay are given, along with the related constraint functions. Details of our proposed incremental relaying strategy is given and compared with the two-relay protocol. The results show that the incremental routing protocol outperforms the two-relay based protocol in terms of network lifetime, throughput. However, the latter protocol could support high traffic load conditions when compared with the former.

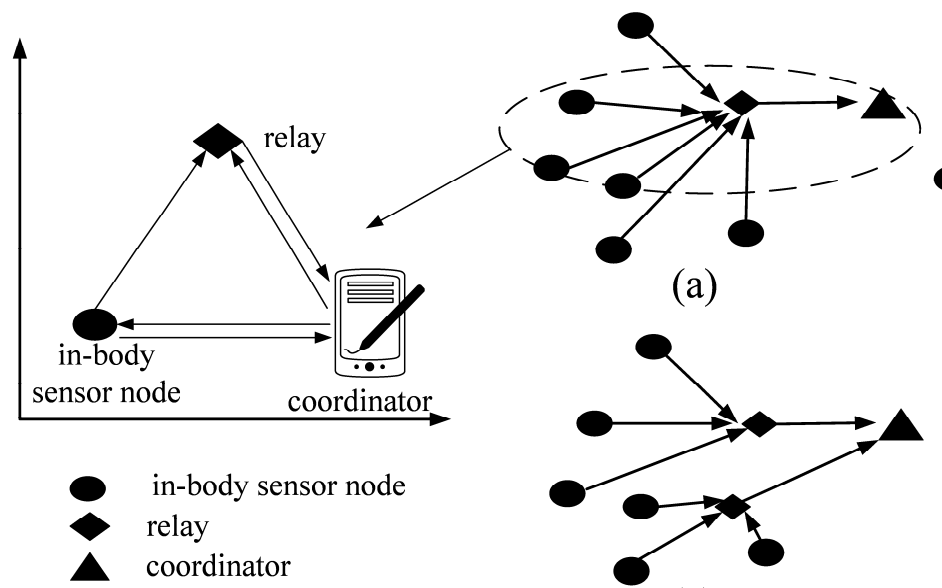

(c)

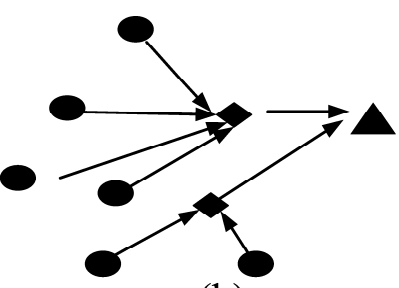

(b)

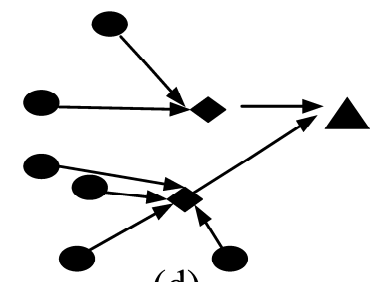

(d)

Figure 10. Demonstration of the relay-based routing protocol; (a) single relay based scenario; (b)-(d) two-relay based selective routing protocols.

\subsection{Radio Model}

The analysis of WBAN system energy consumption is given by extending our previous work on flexible QoS WBANs [3]. Assuming the transmission packet length is defined as $k$, the minimal transmission energy consumption of sensor nodes can be expressed as:

$$
E_{T x \_m i n}(d, k)=k E_{T x_{\text {elec }}}+k E_{\text {amp }} d n
$$

where $E_{T x_{-} \text {min }}$ and $E_{T x_{\_} \text {elec }}$ mean the minimal required energy for data transmission from a sensor node to a relay and the essential energy consumption to activate the electronic circuit, respectively.

The distance between the transmitting sensor node and the receiver side is represented by $d$, and $n$ is the path loss exponent. Similarly, the minimal energy consumption for the reception process can be regarded as $E_{R x \text {, min }}=k E_{\text {elec }}$. The minimal total energy consumption for a sensor node $E_{\text {Total }}$ can be expressed as:

$$
E_{\text {Total }}(k, d)=E_{\text {Tx_min }}(d, k)+k E_{\text {elec }}
$$

The PL parameters in Table 3 are utilized when the in-body sensor nodes transfer data to the relay nodes. Two commercially available WBAN transceivers, the Nordic nRF2401A and Chipcon CC2420 are utilized and the related radio parameters have been summarized in Table 5 [36].

Table 5. Radio parameters of nRF2401A and CC2420.

\begin{tabular}{ccc}
\hline Parameter (Unit) & nRF2401A & CC2420 \\
\hline Tx current $(\mathrm{mA})$ & 10.5 & 17.4 \\
Rx current $(\mathrm{mA})$ & 18 & 19.7 \\
Voltage $(\mathrm{V})$ & 1.9 & 2.1 \\
$E_{T x \_e l e c}(\mathrm{~nJ} / \mathrm{bit})$ & 16.7 & 96.9 \\
$E_{R x \_e l e c}(\mathrm{~nJ} / \mathrm{bit})$ & 36.1 & 172.8 \\
$E_{\text {amp }}\left(\mathrm{nJ} / \mathrm{bit} / \mathrm{m}^{2}\right)$ & 1.97 & 2.71 \\
\hline
\end{tabular}




\subsection{QoS Metric Modeling}

\subsubsection{Network Lifetime}

The stability period and total network lifetime are defined as the lifespan of the network until the first in-body sensor node is energy depleted, including the time duration of the network, until all in-body sensor nodes are energy depleted, respectively [36]. Assuming the number of in-body sensor nodes is $N$ and each node is initially equipped with energy $E_{0}$. The main object of I2O WBAN is to maximize the network lifetime $T$, which can be formulated via linear programing as [21]:

$$
\text { Objective: } \operatorname{Max} T=\sum_{r} t_{r}
$$

where $r$ and $t_{r}$ denotes the current round and summation of rounds before all in-body sensor nodes energy deplete, respectively. The energy consumption per bit consists of sensing, processing and transmitting energy for an in-body sensor node represented by $E_{\text {sen }}, E_{\text {pro }}$ and $E_{\text {trans }}$ [21]. The remaining energy of the I2O WBAN network after each round can be defined as network residual energy $E_{i}$. In terms of constraints, the $\mathrm{I} 2 \mathrm{O}$ WBAN is subject to:

$$
\begin{gathered}
t_{r} \geqslant \frac{E_{i}}{\sum_{i} k\left(E_{\text {sen }}^{i}+E_{\text {pro }}^{i}+E_{\text {trans }}^{i}+n E_{\text {amp }}^{i} d_{S R}\right)}, \forall i \in N \\
E_{o} \geqslant E_{i}, \forall i \in N \\
E_{i} \rightarrow 0, \forall i \in N \\
\sum_{i} f_{S R}>\sum_{r} f_{R C}, \forall i \in N
\end{gathered}
$$

where $f_{R C}$ and $f_{S R}$ represent the data flow directions from relay $R$ to the coordinator $C$ and from in-body sensor node $S$ to the corresponding relay $R$, respectively. The Constraint (17) illustrates the network energy consumption per round. Constraints in (18) and (19) are the energy requirements, the network residual energy $E_{i}$ reduces after each round and is finally exhausted. Constraint (20) demonstrates that the data flow should be transmitted from node $S$ to the coordinator via a corresponding relay $R$. Moreover, violation of (20) leads to heavy traffic conditions resulting in transmission delay and packet dropping.

\subsubsection{Network Throughput}

The network throughput represents the total number of successfully received information packets at the coordinator. It is important to maximize the number of successfully transmitted packets at the coordinator $C$, because all information is critical in I2O WBANs. The optimization expression for maximizing the number of successfully received packets $P_{S}$ can be formulated as:

$$
\text { Objective : } \operatorname{Max} \sum_{r} P_{s}, \forall r \in T
$$

Subject to:

$$
\begin{gathered}
P_{S R}>P_{R C}, \forall S \in N, \forall R \in N \\
E_{i} \geqslant E_{T x \_ \text {min }} \\
P_{\text {link }} \geqslant P_{\text {min }}
\end{gathered}
$$

The objective function (21) aims to maximize the number of successfully received packets $P_{S}$ during the network lifetime T. Constraint (22) demonstrates that data packets may drop when data transmission occurs from $R$ to $C$. Constraint (23) points out that no data information transmission is possible when the remaining energy $E_{i}$ is lower than the minimal required transmission energy 
$E_{T x_{-} \min }$ as mentioned in (14). Constraint (24) states that the probability of a transmission link $P_{\text {link }}$ should be no less than the minimal predetermined required value $P_{\min }$.

\subsubsection{Delay}

As analyzed in (17) and (20), maximizing the network lifetime will increase the delay. In addition, the I2O WBAN links suffer from high energy attention leading to transmission link instability causing higher data transmission delay. Propagation delay is an important factor in dealing with high data rate transmission scenarios. The mathematical model of the end to end delay can be expressed as:

$$
\text { Objective: } \operatorname{Min} \tau_{S C}=\tau_{S}+\tau_{R C}
$$

where $\tau_{S C}$ is the delay for the in-body node $S$ transmits to the coordinator $C$. $\tau_{S}$ and $\tau_{R C}$ represent nodal delay at $S$ and delay for data transmission between $R$ and $C$, respectively.

Subject to:

$$
\begin{gathered}
\tau_{S} \geqslant \tau_{S}^{T x}+\tau_{S}^{\text {queue }}+\tau_{S}^{\text {Proc }}+\tau_{S}^{C C}, \forall S \in N \\
x \geqslant N \geqslant 0, \forall x \in Z^{+} \\
P_{S R} \geqslant P_{S} \\
\gamma_{S}^{\text {dep }} \geqslant \gamma_{S}^{\text {arr }} \\
B E R_{i} \geqslant B E R_{\text {pre }} \\
\text { Min } d_{S R} \rightarrow d_{\text {min }}
\end{gathered}
$$

Constraint (26) illustrates that the nodal delay $\tau_{S}$ consists of the propagation delay $\tau_{S}^{T x}$, queuing delay $\tau_{S}^{q u e u e}$, data processing delay $\tau_{S}^{\text {Proc }}$ and channel capture delay $\tau_{S}^{C C}$ [21]. Constraint (27) provides the upper and lower bounds for $N$. Considering a dense I2O WBAN network, (number of $N$ is very large), the channel access delay $\tau_{S}^{C C}$ will increase due to more sensor nodes contending the channel access. Constraint (28) regulates the number of data packets that need optimizing due to the limited packet handing capacity at the receiver. Similarly, Constraint (29) demonstrates that the packet arrive rate $\gamma_{S}^{\text {arr }}$ should be less than the packet transmit rate $\gamma_{S}^{d e p}$ and this can reduce the queuing delay $\tau_{S}^{q u e u e}$. Constraint (30) points out that BER should be higher than a predetermined BER threshold, otherwise the rate of dropped packets will increase $\tau_{S}^{\text {Proc }}$. Constraint (31) illustrates that minimizing the transmission distance is an effective method to decrease the propagation delay $\tau_{S}^{T x}$, which can be defined as:

$$
\tau_{S}^{T x}=d(S) / c
$$

where $d(S)$ is the distance between the in-body sensor node to relay in each round and $c$ is the speed of electromagnetic wave, respectively. Since there is no agreement or standard for superframe structures in $\mathrm{I} 2 \mathrm{O}$ WBAN devices, we follow [37] and estimate the delay as stated in (32).

\subsection{Proposed Protocols}

The overall flow chart for the protocol is shown in Figure 11 and we now give details of the various operations within it. 


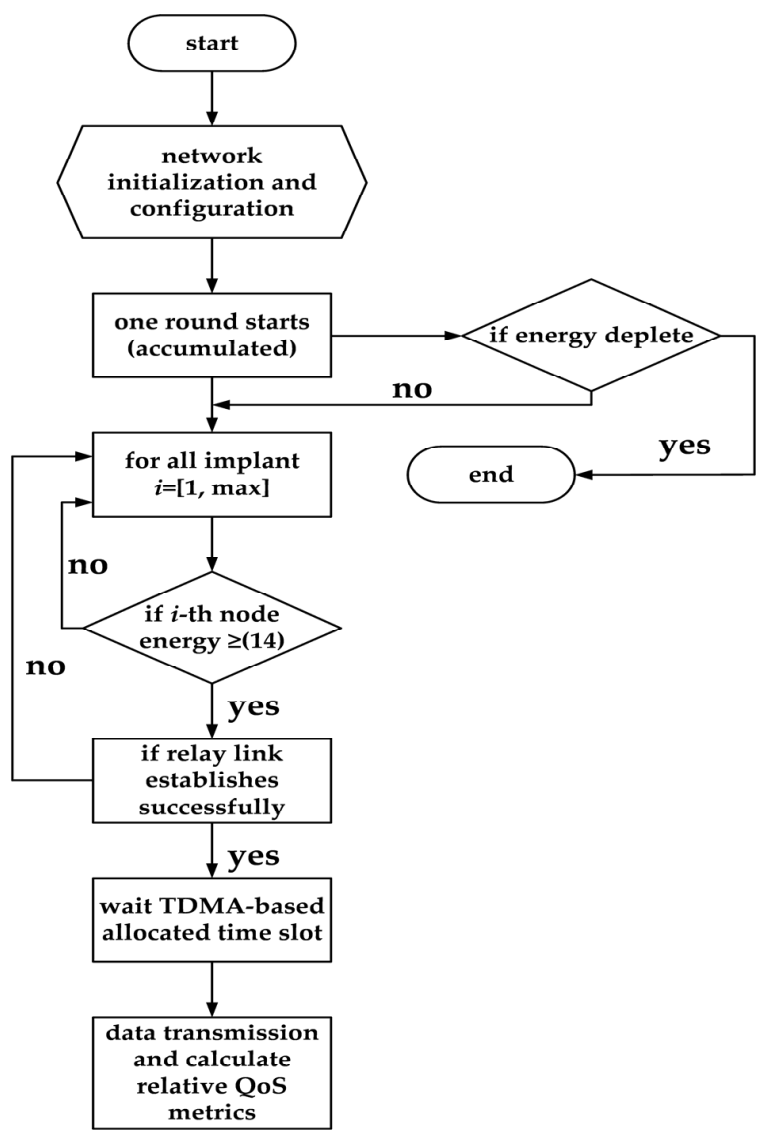

Figure 11. The information flow for the proposed protocol.

\subsubsection{Initialization Phase}

All in-body sensor nodes and relays are assigned a unique ID. The data transmission time division multiple access (TDMA) schedules for sensors and relays are based on those IDs. The first round starts once the network initialization and configuration have finished. The coordinator checks the energy status of all in-body sensor nodes.

\subsubsection{Routing Phase}

If the in-body sensor node residual energy is greater than (14), then the coordinator will check the distance between a relay and the node. The coordinator assigns TDMA time slots to the in-body sensor node and a nearby relay, which is selected based on the cost function similarly to [36]:

$$
C(S)=d(S) / R(S)
$$

Relay nodes are available for all in-body sensor nodes. For the two-relay based protocol, when two possible relays have the same cost function values, the one with a smaller value of (15) is selected.

\subsubsection{Transmission Phase}

In the data transmission scheduling stage, relays assign TDMA based allocated time slots to the in-body sensor nodes. A communication link between the selected in-body sensor node and the nearby relay is established. The selected in-body sensor node transfers the sensed data during the allocated time slot to the nearby relay node. The relay node receives the data from the in-body sensor node and forwards them to the coordinator during the allocated time slots. The process will continue with the number of rounds accumulating until the energy of all the in-body sensor nodes is depleted. 


\subsection{Performance Evaluation and Results}

The topology is introduced as follows, a coordinator is located in the center of the human body and six in-body sensor nodes are also positioned within the body. The coordinates of the coordinator and the in-body sensor nodes are summarized in Table 6. All in-body nodes have the same initial energy of 0.5 Joules. The number of relays is limited to two with coordinates $(1.65,0.75)$ and $(0.9,1.65)$. As presented in (24), a probabilistic approach (random uniform model) with a probability of packet loss of 0.3 is utilized in all simulation cases in agreement with [36]. The packet size is set as 2000 bits, which is defined as the maximum payload based on the IEEE 802.15.6 standard [22]. Simulation parameters are summarized in Table 7.

Table 6. The Coordinates of In-Body Nodes and Coordinator.

\begin{tabular}{ccc}
\hline Node ID & X-Coordinate & Y-Coordinate \\
\hline 1 & 0.2 & 1.6 \\
2 & 0.4 & 0.4 \\
3 & 0.3 & 0.1 \\
4 & 0.6 & 0.35 \\
5 & 0.7 & 1.5 \\
6 & 0.9 & 1.65 \\
coordinator & 0.45 & 0.85 \\
\hline
\end{tabular}

Table 7. Simulation Parameters.

\begin{tabular}{cc}
\hline Simulation Parameter (Unit) & Value \\
\hline Number of in-body nodes & 6 \\
Network Initial energy (Joule) & 3 \\
Payload size (bits) & 2000 \\
Electromagnetic wave speed (m/s) & $3 \times 10^{8}$ \\
Packet loss probability & 0.3 \\
\hline
\end{tabular}

Figure 12 illustrates the comparison of the stable period and total network lifetime for the proposed protocols. It can be seen that the incremental relay-based routing protocol achieves a longer stability period and total network lifetime than the two-relay based protocols with two different transceivers. This is because the second relay node only receives and forwards the data from the in-body sensor nodes when the first relay fails and therefore minimizes the network energy consumption. The stability periods of the incremental relay-based protocol are circa 4000 rounds using the using nRF2401A and circa 1500 rounds with the CC2420, whereas the two-relay based delivers 3400 rounds and 1200 rounds, respectively.

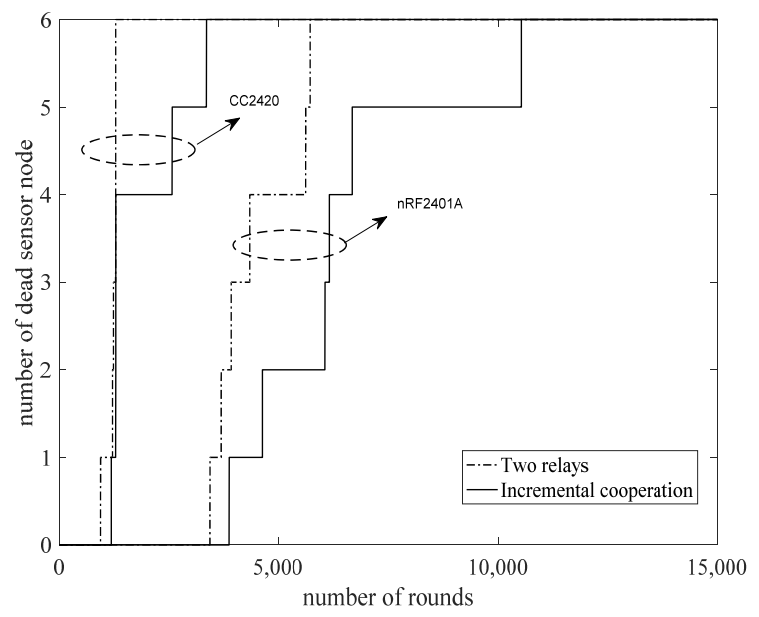

Figure 12. The relationship berween the number of dead nodes and network lifetime. 
Figure 13 presents the comparison of network residual energy of two protocols for the two transceivers considered. The nRF2410A-based incremental relay-based protocol has the highest network residual energy and thus leads to the longest network lifetime. Figure 14 demonstrates that the average energy consumption per round of the incremental protocol is $0.27 \mathrm{~mJ}$ and $0.57 \mathrm{~mJ}$ when deploying nRF2401A and CC2420, which achieves nearly 52\% and 45\% less energy consumption when compared to the two-relay based protocol. Reasons for the above results are as follows: (a) relay nodes forward data even if when it is not necessary, resulting in channel resouce waste; (b) an alternative data transmission link only starts to work when the first link fails.

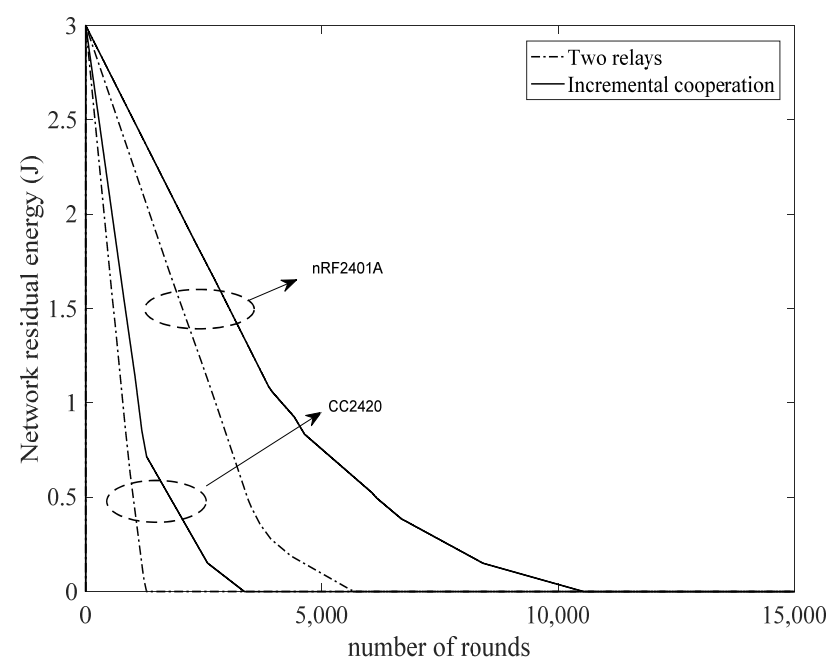

Figure 13. The residual energy versus the network lifetime.

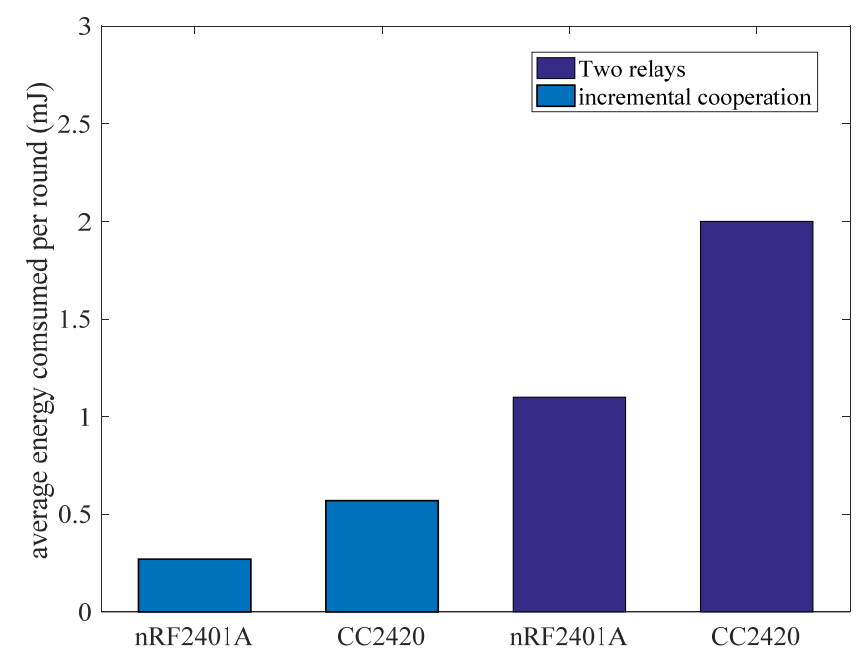

Figure 14. Average energy consumption per round.

Data loss is usually caused by transmission attenuation and shadow fading as presented in Section 3 [21]. Figure 15 shows the number of successfully transmitted information packets in the network and Figure 16, the network delay. It can be seen that the two-relay protocol can promise higher data transmission rates than the incremental relay-based protocol when deploying the same transceiver. The number of total transmission packets depends on the number of alive, in-body sensor nodes and the total network lifetime. The shorter lifetime of the two-relay routing technique is the major factor in its decreased throughput. The propagation delay depends on the overall I2O WBAN communication distance. Moreover, it can be seen from Figures 15 and 16 that more information packets generated by the system leads to higher propagation delays in the network. 


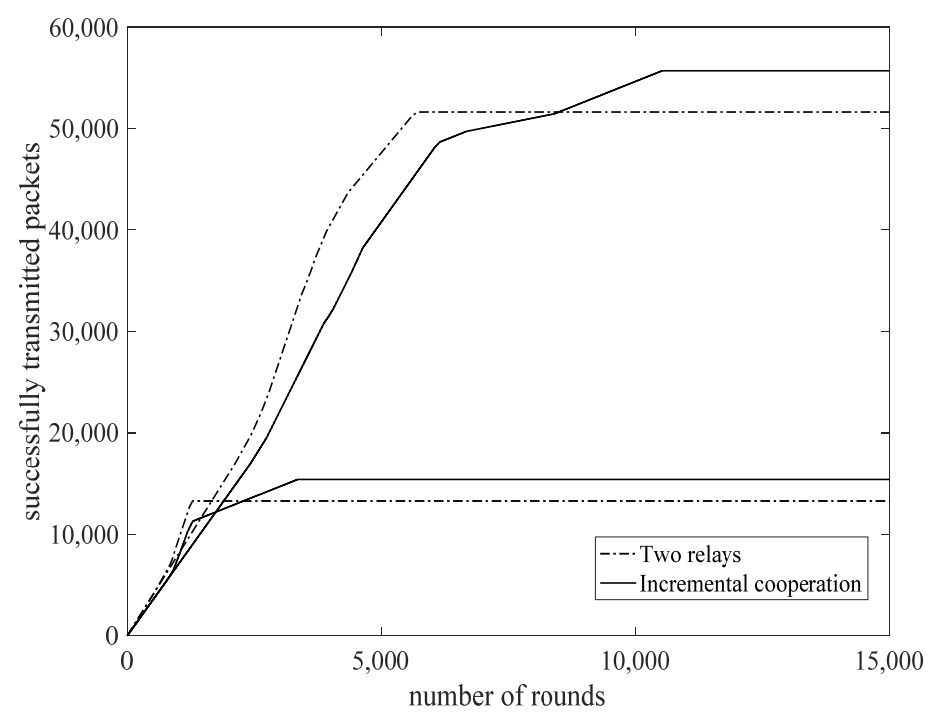

Figure 15. The successfully transmitted packets versus the network lifetime.

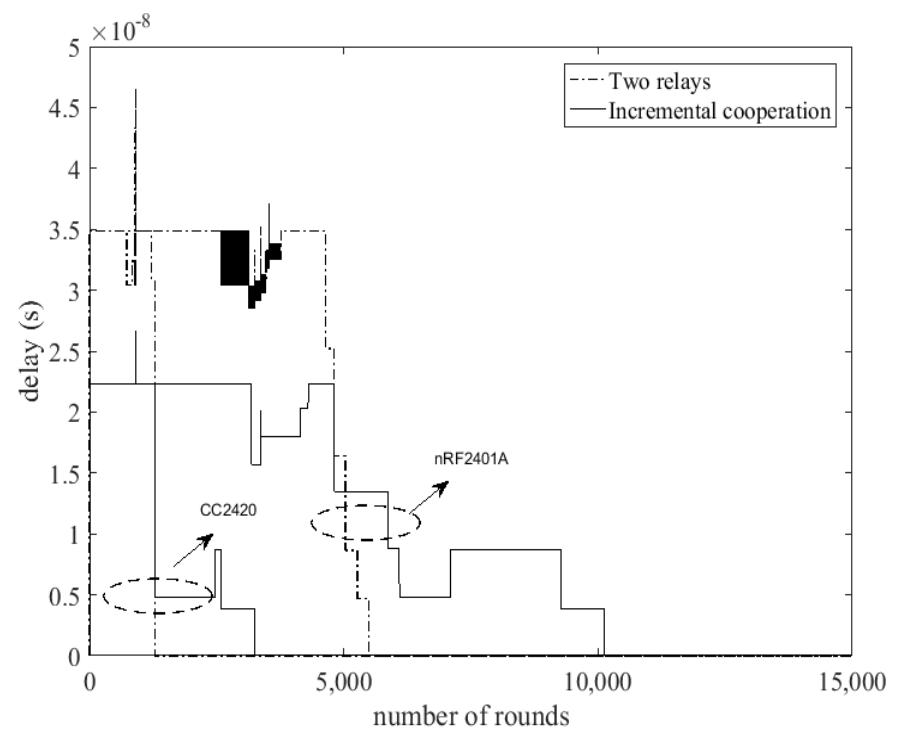

Figure 16. The propagation delay versus the network lifetime.

For related QoS metrics, the results show that the nRF2401A incremental technique can achieve a longer stability period and network lifetime, minimizes average network energy consumption and delivers the highest number of successfully transmitted packets. It is therefore a promising technique in the support of long-time healthcare services. For high data rate transmission or heavy traffic load requirement applications, the two-relay based approach would achieve better performance. Moreover, we compare the attributes of the other state of art existing routing techniques with our proposed protocol as summarized in Table 8. Further study will be required in order to optimise energy efficient routing protocols and to ensure data packet IP compatibility, both of which are considered essential for the ongoing health of IoT applications. 
Table 8. Comparison of the Proposed Protocol With Other State of Art Techniques [21,34,37].

\begin{tabular}{|c|c|c|c|}
\hline Protocol & Features & Weaknesses & Performance \\
\hline $\begin{array}{l}\text { Incremental relaying } \\
\text { (this paper) }\end{array}$ & $\begin{array}{l}\text { - Cooperative routing } \\
\text { - Considering multiple QoS metrics } \\
\text { - Cost function to select route } \\
\text { - Probabilistic approach }\end{array}$ & - Not suitable for heavy load traffic & $\begin{array}{l}\text { - Extend network lifetime } \\
\text { - Higher throughput } \\
\text { - Minimize energy consumption } \\
\text { - Reduce propagation delay }\end{array}$ \\
\hline $\begin{array}{l}\text { Energy-Balanced Rate } \\
\text { Assignment and Routing } \\
\text { protocol (EBRAR) }\end{array}$ & $\begin{array}{l}\text { - Routing strategy based on the residual } \\
\text { energy of sensors } \\
\text { - Intelligent transmission routes } \\
\text { - Balance payload on sensors }\end{array}$ & $\begin{array}{l}\text { - low bandwidth utilization } \\
\text { - High drop packets } \\
\text { - High delay }\end{array}$ & $\begin{array}{l}\text { - Minimum energy consumption } \\
\text { - Decrease packet loss } \\
\text { - Fault resistance }\end{array}$ \\
\hline $\begin{array}{l}\text { Enhanced cooperative } \\
\text { critical data transmission } \\
\text { in emergency in static } \\
\text { WBAN (EInCo-CEStat) }\end{array}$ & $\begin{array}{l}\text { - Incremental relaying } \\
\text { - Three relays } \\
\text { - Cooperation communication }\end{array}$ & $\begin{array}{l}\text { - Decrease stability period } \\
\text { - Low energy efficiency }\end{array}$ & $\begin{array}{l}\text { - Very high throughput } \\
\text { - Improve packet error rate }\end{array}$ \\
\hline $\begin{array}{l}\text { Link-Aware and Energy } \\
\text { Efficient protocol for } \\
\text { WBANs (LAEEBA) }\end{array}$ & $\begin{array}{l}\text { - Minimize path loss } \\
\text { - Cost function to select the routine } \\
\text { - Support emergency data transmission }\end{array}$ & $\begin{array}{l}\text { - Throughput is not satisfactory } \\
\text { - Only consider on-body } \\
\text { communication channels }\end{array}$ & $\begin{array}{l}\text { - Limited stability period } \\
\text { - Limited network lifetime } \\
\text { - Energy efficiency }\end{array}$ \\
\hline $\begin{array}{l}\text { Relay based routing } \\
\text { protocol for in-body } \\
\text { sensor networks }\end{array}$ & $\begin{array}{l}\text { - Energy efficient } \\
\text { - Improve faults tolerant performance }\end{array}$ & $\begin{array}{l}\text { - Path loss model is not accurate } \\
\text { - Limited throughput }\end{array}$ & $\begin{array}{l}\text { - Prolong the network lifetime } \\
\text { - Energy efficient }\end{array}$ \\
\hline
\end{tabular}




\section{Discussion and Open Research Issues}

In this section, we report and analyze the QoS mechanism, target-specific QoS requirements and the most relevant QoS factors for service systems. Some emergency issues and future research topics in I2O WBAN system are also listed. The requirements and potential architecture of the health of IoT based I2O WBANs is discussed.

\subsection{QoS in I2O WBANs}

\subsubsection{Candidate Radio Technologies in I2O WBANs}

An I2O WBAN is a type of wireless body area sensor network (WBASN) and centers on the human intra-body region using low power consumption [3,9]. There are several potential communication techniques in this area such as Bluetooth, WiFi, Zigbee and near field communication (NFC) [4]. Moreover, wireless coexistence communication technologies in I2O WBAN, such as LP-WiFi, are also promising candidates for future research [38].

\subsubsection{QoS Metrics for I2O WBANs}

Despite significant developments in wireless communication technology, QoS handling for different healthcare applications in I2O WBANs remains a challenging issue $[39,40]$. The general QoS metrics from the I2O WBAN network perspective involve network throughput, reliability transmission, energy efficiency, network lifetime and so on. It should be noticed that applications may request target-specific QoS support by specifying their requirements in terms of one or more of the QoS metrics. In general, an I2O WBAN is required to analyze the application requirements and deploy various QoS mechanisms [35]. Table 9 illustrates the QoS mechanism solutions based on requirements [4,41].

Table 9. QoS Mechanism Solutions Based on Requirements.

\begin{tabular}{ccccc}
\hline QoS Mechanism & Reliability & Real-Time Transmission & Energy Efficiency & Adaptability \\
\hline Data collision & - & $\sqrt{ }$ & $\sqrt{ }$ & $\sqrt{ }$ \\
Data compression & - & - & $\sqrt{ }$ & $\sqrt{ }$ \\
Error control coding & $\sqrt{ }$ & $\sqrt{ }$ & $\sqrt{ }$ & $\sqrt{ }$ \\
Power control & $\sqrt{ }$ & $\sqrt{ }$ & - & $\sqrt{ }$ \\
Targeted ability & - & - & & \\
\hline
\end{tabular}

\subsubsection{QoS Requirements for $\mathrm{I} 2 \mathrm{O}$ WBANs}

Optimization of communication systems that can realise the target-specific health IoT QoS requirements involves multiple factors [1]. As mentioned above, for transplanted organs, the battery lifetime of the implanted devices would be the crucial factor, with the data transmission rate not the primary concern. For diabetic patient implanted devices, a reliable transmission channel for glucose data transmission would be the vital issue and thus may require significant energy consumption to improve channel quality (e.g., a higher predetermined BER may be required). Surgical operations, such as wireless capsule endoscopes and biomedical image processing need relatively high data transfer speeds (nearly $10 \mathrm{Mbps}$ ) and our proposed $\mathrm{I} 2 \mathrm{O}$ communication systems are able to reliably support these up to a few meters. The key factors of the selected in-body and on-body WBAN applications discussed in this paper are summarized in Table $10[8,9,42]$.

Table 11 highlights the QoS requirements of WBAN applications [39]; Table 12 demonstrates detailed QoS parameters of WBAN service requirements in the application, transport, network, media access control (MAC) and physical layers [4,39]. Thus, I2O WBAN and system service QoS issue metrics and requirements in relation to each layer, are summarised and analysed in Tables 9-12. 
Table 10. The Key Factors of Selected In-Body and On-Body WBAN Applications $[6,7,20]$.

\begin{tabular}{|c|c|c|c|c|c|c|}
\hline Application & Sensor & Energy Consumption & BER & Operating Distance & Lifetime & Data Rate \\
\hline \multirow{2}{*}{ On-body applications } & ECG & Low & Low & High & $>1$ week & $>3 \mathrm{Kbps}$ \\
\hline & Blood pressure & Low & Low & Low & Very long & $<10 \mathrm{Kbps}$ \\
\hline \multirow{5}{*}{ In-body applications } & ICD & Moderate & High & Low & $>40 \mathrm{~h}$ & Few Kbps \\
\hline & Organ monitoring & Low & Moderate & Moderate & 7-10 days & $>100 \mathrm{Kbps}$ \\
\hline & Glucose & High & Moderate & Low & $>1$ week & Few Kbps \\
\hline & Capsule endoscope & High & High & Moderate & $>24 \mathrm{~h}$ & $\sim 10 \mathrm{Mbps}$ \\
\hline & Image processing & High & High & Low & $>12 \mathrm{~h}$ & $\sim 10 \mathrm{Mbps}$ \\
\hline
\end{tabular}

Table 11. The QoS Requirement of WBAN Applications [20,21].

\begin{tabular}{|c|c|}
\hline QoS Requirement & WBAN \\
\hline Data rate & WBAN communication systems should cover bit rates from few Kbps to $\sim 30 \mathrm{Mbps}$ \\
\hline Tolerance & Stand $\sim 3 \mathrm{~s}$ when sensor nodes either added or removed \\
\hline Maximum number of sensor nodes & $\leqslant 256$ \\
\hline Mobility & $\begin{array}{c}\text { Capable to reliable transmission when people moving } \\
\text { Data should not loss even if capacity is reduced } \\
\text { Anti-interference when people moving }\end{array}$ \\
\hline Latency & $\begin{array}{l}\text { Latency }<125 \mathrm{~ms} \text { for medical applications, Latency }<250 \mathrm{~ms} \text { for non-medical service } \\
\text { Jitter }<50 \mathrm{~ms} \text { for all applications }\end{array}$ \\
\hline Coexistence & In-body and on-body sensor nodes should able to work together \\
\hline
\end{tabular}


Table 12. Important QoS Parameters of WBAN Service Systems [4,21].

\begin{tabular}{|c|c|c|c|}
\hline Layer & QoS Issues & QoS Metric & QoS Requirements \\
\hline Application & $\begin{array}{l}\text { - Total system lifetime } \\
\text { - Data fusion ability } \\
\text { - Error tolerant }\end{array}$ & $\begin{array}{l}\text { - Communication distance } \\
\text { - Resource allocation } \\
\text { - Available working time } \\
\text { - Fault awareness }\end{array}$ & $\begin{array}{l}\text { - Maximum system lifetime } \\
\text { - Transmission reliability } \\
\text { - Security }\end{array}$ \\
\hline Transport & $\begin{array}{l}\text { - Link reliability } \\
\text { - Latency } \\
\text { - Packet loss } \\
\text { - Transmission corruption }\end{array}$ & $\begin{array}{l}\text { - Delay } \\
\text { - Jitter (delay variance) } \\
\text { - Buffer } \\
\text { - Error packet ratio } \\
\text { - Energy efficient strategy }\end{array}$ & $\begin{array}{l}\text { - Minimum transmission latency } \\
\text { - Minimum energy consumption } \\
\text { - Decrease packet loss } \\
\text { - Suitable data rates awareness } \\
\text { - Fault resistance }\end{array}$ \\
\hline Network & $\begin{array}{l}\text { - Path latency } \\
\text { - Routing } \\
\text { - Mobility capability } \\
\text { - Faults tolerant }\end{array}$ & $\begin{array}{l}\text { - Channel latency } \\
\text { - Traffic strategy } \\
\text { - System robustness }\end{array}$ & $\begin{array}{l}\text { - Minimise latency } \\
\text { - Mobility support } \\
\text { - Routing fault tolerance } \\
\text { - Energy control (lower overhead) }\end{array}$ \\
\hline MAC & $\begin{array}{l}\text { - Throughput } \\
\text { - Delay } \\
\text { - Packets delivery } \\
\text { - Collision management } \\
\text { - Faults tolerant } \\
\text { - Power management }\end{array}$ & $\begin{array}{l}\text { - Data rate } \\
\text { - Throughput } \\
\text { - Collision probability } \\
\text { - interference immunity } \\
\text { - Bandwidth utilisation } \\
\text { - link fault tolerant }\end{array}$ & $\begin{array}{l}\text { - Improve reliability } \\
\text { - Error control (channel coding) } \\
\text { - Minimise data collision } \\
\text { - Decrease interference }\end{array}$ \\
\hline Physical & $\begin{array}{l}\text { - Topology } \\
\text { - Physical standards (such as interface) } \\
\text { - Bandwidth management } \\
\text { - } \mathrm{Tx}(\mathrm{s}), \operatorname{Rx}(\mathrm{s}) \text { faults tolerant }\end{array}$ & $\begin{array}{l}\text { - Capacity } \\
\text { - Data rate } \\
\text { - SNR } \\
\text { - Power attenuation }\end{array}$ & $\begin{array}{l}\text { - Efficient topology } \\
\text { - Data rate } \\
\text { - Priority information guarantee } \\
\text { - Channel allocation strategy }\end{array}$ \\
\hline
\end{tabular}




\subsection{Emerging I2O WBAN Issues}

\subsubsection{I2O WBAN Packet Design}

To incorporate the diverse QoS requirements of heterogeneous communication networks into a packet format, superframe structures are promising technologies that allow $\mathrm{I} 2 \mathrm{O}$ devices with a particular traffic type to transmit during the period that is best suited in meeting the corresponding QoS [42]. Here, we assume that this aspect is in existence, whilst acknowledging that there is further work to be done in order to bring it into routine practice.

\subsubsection{I2O WBAN Interface Design}

The in-body environment makes I2O WBAN technologies more complex and challenging when compared with other communication networks. I2O WBAN systems can be regarded as a 'shared bus', where different kinds of entities that can generate data and transfer the data provided by other entities [43-46]. Recently, software-based results related to WBAN entity interfaces design have been presented [44]. However, the in-body WBAN interface design should consider both network configuration and network management and there is to date no agreed international standard for the I2O interface technologies [43,44]. Different layer requirements for in-body device (logical management) entities interface, are reported in [40]. One promising solution reported in [44], is a radio-based $\mathrm{I} 2 \mathrm{O}$ system that enables data transmission between the in-body device(s) to the e-health gateway via an external programming device. For on-body WBAN interface technologies, smartphones and other on-body sensor nodes (wearable devices) with data collection, data buffering, information transmission, user authentication, computational and communication capabilities have been proposed to work together with the in-body interface [3,47].

\subsubsection{I2O WBAN Models Validation}

Owing to technical constraints and legal provisions, practical human $\mathrm{I} 2 \mathrm{O}$ radio channel experiments are not possible [48,49]. Alternative approaches to validate the I2O WBAN communication systems involve advanced computational electromagnetics and biological phantoms $[5,49]$. In the future, multi-disciplinary collaboration with clinicians to measure radio channel in animals is one promising technique that can derive statistical $\mathrm{I} 2 \mathrm{O}$ body models that could overcome the limitations of performing measurements in humans.

\subsection{Analysis of I2O WBAN Based Health IoT}

An effective approach for the interconnection of WBAN systems is to use the Internet Protocol (IP) [35]. WBAN information packets can be processed and translated into IP datagrams by a gateway or a smartphone on various available platforms, such as the Advanced Health and Disaster Aid Network (AID-N) and the Microsystems Platform for Mobile Services and Applications (MIMOSA) [20]. Smartphones equipped with multiple network interfaces could enable the user to interact with the linked WBAN and forward data to physicians in any location [35,50].

To date, the core of health IoT solutions is low power, wireless personal area networks (6LoWPAN) and Internet Protocol for Smart Objects (IPSO), which are predicted by the Internet Engineering Task Force (IETF) and aim to manage WBAN devices Internet connectivity issues through IP version 6 (IPv6) [35]. It is of great significance that the I2O WBAN infrastructure supports access to the health IoT. Meanwhile, more effort should be spent on I2O WBANs low power consumption routing protocol design in order to minimize the energy consumption and improve multiple QoS metric performance. An I2O WBAN sensor address configuration process should consider uniqueness, low energy consumption and offer address reclamation. Once a WBAN system collects the physical parameters of a human body, all sensor nodes in the WBAN must be configured with a unique address [48]. In recent years, the IEEE and other authorities have standardized numerous protocols to support WBANs [20]. However at present, the proposed standardized protocols are ineligible for 
an I2O WBAN [20,35,51]. For example, the IEEE 802.15.3 standard is designed for high data speed, wireless personal area networks (WPANs) that can reach up to $20 \mathrm{Mbps}$. However, this standard does not support energy efficiency and other QoS requirements [35]. The IEEE 802.15.4 standard is considered as an energy efficient protocol but only capable for low data rate applications and services [35]. The latest IEEE 802.15.6 defines MAC and PHY layers for low-power consumption implant devices, whereas the security requirements of data authentication and encryption have not been well defined [20]. The Zigbee IP is the first open standard for the IPv6 standard, which enriches the WBAN services by adding network and security layers. However, recently, the Zigbee Alliance decided to incorporate standards from IETF into its technical specifications [20].

I2O WBANs can be divided into two categories; (1) every sensor node is provided with a unique entity or; (2) the network is an entity and accessible via a coordinator node that has full information about the network [50]. I2O WBAN sensor nodes are attached on, or within the human body area to process sensed data with wireless transfer to a coordinator connected to the internet [3]. Considering QoS-aware requirements, this paper presents a potential QoS-aware protocol design for I2O WBAN with the following characteristics:

(1) The I2O WBAN consists of at least one full function device (such as a smartphone) and a series of reduced function small-size sensor nodes, which are assigned a unique ID.

(2) Each sensor node senses different physical parameters to reduce the address configuration cost.

(3) A relay strategy is considered, which decreases system configuration delays and minimizes the overall length of communication distance within I2O WBAN.

\section{Conclusions}

In this paper, we first propose and analyze an efficient and accurate PL model for the 3D human $\mathrm{I} 2 \mathrm{O}$ communication system at $2.45 \mathrm{GHz}$. Due to the limitation of the implanted sensor batteries, we have investigated and compared several established high efficiency modulation schemes. The threshold SNRs of BPSK, QPSK, 16PSK and 16QAM are approximately $11 \mathrm{~dB}, 13 \mathrm{~dB}$ and $15.5 \mathrm{~dB}$ and $18 \mathrm{~dB}$ respectively, when an acceptable predetermined BER of $10^{-3}$ is adopted. Results demonstrate that the communication system can achieve satisfactory performance at relatively high data rates of $30 \mathrm{Mbps}$ over distances of up to $1.6 \mathrm{~m}$. Alternatively it can provide an highly reliable communication link for longer distances, at lower data rates $(0.25$ to $5 \mathrm{Mbps})$ by adopting the BPSK modulation technique. Based on the proposed I2O WBAN system, an energy efficient incremental routing protocol has been implemented and compared with the existing two-relay strategy. Simulation results demonstrate that our proposed data routing technique could significantly improve the performance of network lifetime, throughput and propagation delay. Open issues and standardization within the I2O WBAN area are summarized and explored as an inspiration towards developments in health IoT applications. Our future work involves radio channel measurements in biological phantoms to validate the PL model and communication system performance. Moreover, this can be extended to animal studies by multi-disciplinary collaboration with clinical professionals and biologists in order to overcome the difficulties of performing measurements in humans.

Author Contributions: This paper is completed by Yangzhe Liao and Chenyao Bai under the guidance of supervisors Mark S. Leeson and Matthew D. Higgins. Yangzhe Liao and Chenyao Bai conceived and designed the research topics and analyzed the data. Yangzhe Liao wrote the paper under the help of Mark S. Leeson.

Conflicts of Interest: The authors declare no conflicts of interest.

\section{References}

1. Islam, S.M.R.; Kwak, D.; Kabir, M.H.; Hossain, M.; Kwak, K.S. The internet of things for health care: A comprehensive survey. IEEE Access 2015, 3, 678-708. [CrossRef]

2. Fan, Y.J.; Yin, Y.H.; Xu, L.D.; Zeng, Y.; Wu, F. IoT-based smart rehabilitation system. IEEE Trans. Ind. Inform. 2014, 10, 1568-1577. 
3. Liao, Y.; Leeson, M.S.; Higgins, M.D. Flexible quality of service model for wireless body area sensor networks. IET Healthc. Technol. Lett. 2016, 3, 12-15. [CrossRef] [PubMed]

4. Movassaghi, S.; Abolhasan, M.; Lipman, J.; Smith, D.; Jamalipour, A. Wireless body area networks: A survey. IEEE Commun. Surv. Tutor. 2014, 16, 1658-1686. [CrossRef]

5. Liao, Y.; Leeson, M.S.; Higgins, M.D. A communication link analysis based on biological implant wireless body area networks. Appl. Comput. Electromagn. Soc. J. 2016, 31, 619-628.

6. Galluccio, L.; Melodia, T.; Palazzo, S.; Santagati, G.E. Challenges and implications of using ultrasonic communications in intra-body area networks. In Proceedings of the 9th Annual Conference on Wireless On-demand Network Systems and Services (WONS), Courmayeur, Italy, 9-11 January 2012.

7. Fang, Q.; Lee, S.; Permana, H.; Ghorbani, K.; Cosic, I. Developing a wireless implantable body sensor network in MICS band. IEEE Trans. Inform. Technol. Biomed. 2011, 15, 567-576. [CrossRef] [PubMed]

8. IEEE standard for safety levels with respect to human exposure to radio frequency electromagnetic fields, $3 \mathrm{KHz}$ to $300 \mathrm{GHz}$ amendment 1: Specifies ceiling limits for induced and contact current, clarifies distinctions between localized exposure and spatial peak power density. Available online: http:/ /ieeexplore.ieee.org/ servlet/opac?punumber=10830 (accessed on 11 July 2016).

9. The International Commission on Non-Ionizing Radiation Protection. Guidelines for limiting exposure to time-varying electric, magnetic, and electromagnetic fields (up to $300 \mathrm{GHz}$ ). Health Phys. 1998, 74, 494-522.

10. Ntouni, G.D.; Lioumpas, A.S.; Nikita, K.S. Reliable and energy-efficient communications for wireless biomedical implant systems. IEEE J. Biomed. Health Inform. 2014, 18, 1848-1856. [CrossRef] [PubMed]

11. Darwish, A.; Hassanien, A.E. Wearable and implantable wireless sensor network solutions for healthcare monitoring. Sensors 2011, 11, 5561-5595. [CrossRef] [PubMed]

12. Elias, J. Optimal design of energy-efficient and cost-effective wireless body area networks. Ad Hoc Netw. 2014, 13, 560-574. [CrossRef]

13. Zhu, S.; Langley, R. Dual-band wearable textile antenna on an EBG substrate. IEEE Trans. Antennas Propag. 2009, 57, 926-935. [CrossRef]

14. Kurup, D.; Joseph, W.; Vermeeren, G.; Martens, L. Path loss model for in-body communication in homogeneous human muscle tissue. Electron. Lett. 2009, 45, 453-454. [CrossRef]

15. Kurup, D.; Joseph, W.; Vermeeren, G.; Martens, L. In-body path loss model for homogeneous human tissues. IEEE Trans. Electromagn. Compat. 2012, 54, 556-564. [CrossRef]

16. Computer Simulation Technology. Available online: https://www.cst.com/ (accessed on 4 April 2016).

17. Roman, K.L.L.; Vermeeren, G.; Thielens, A.; Joseph, W.; Martens, L. Characterization of path loss and absorption for a wireless radio frequency link between an in-body endoscopy capsule and a receiver outside the body. EURASIP J. Wirel. Commun. Netw. 2014, 2014, 1-10.

18. Hannan, M.A.; Abbas, S.M.; Samad, S.A.; Hussain, A. Modulation techniques for biomedical implanted devices and their challenges. Sensors 2012, 12, 297-319. [CrossRef] [PubMed]

19. Elhadj, H.B.; Chaari, L.; Kamoun, L. A survey of routing protocols in wireless body area networks for healthcare applications. Int. J. E Health Med. Commun. 2012, 3, 1-18. [CrossRef]

20. Cao, H.; Leung, V.; Chow, C.; Chan, H. Enabling technologies for wireless body area networks: A survey and outlook. IEEE Commun. Mag. 2009, 47, 84-93. [CrossRef]

21. Javaid, N.; Ahmad, A.; Khan, Y.; Khan, Z.A.; Alghamdi, T.A. A relay based routing protocol for wireless in-body sensor networks. Wirel. Personal Commun. 2015, 80, 1063-1078. [CrossRef]

22. Deepak, K.; Babu, A.V. Improving energy efficiency of incremental relay based cooperative communications in wireless body area networks. Int. J. Commun. Syst. 2015, 28, 91-111. [CrossRef]

23. Kurup, D.; Vermeeren, G.; Tanghe, E.; Joseph, W.; Martens, L. In-to-out body antenna-independent path loss model for multilayered tissues and heterogeneous medium. Sensors 2015, 15, 408-421. [CrossRef] [PubMed]

24. Dielectric Properties of Body Tissues. Available online: http://niremf.ifac.cnr.it/tissprop/ (accessed on 29 March 2016).

25. Kurup, D.; Joseph, W.; Vermeeren, G.; Martens, L. Specific absorption rate and path loss in specific body location in heterogeneous human model. Microwaves. IET Antennas Propag. 2013, 7, 35-43. [CrossRef]

26. Liu, X.; Hin, T.K.; Heng, C.H.; Gao, Y.; Toh, W.D.; Cheng, S.J.; Je, M. A 103 pJ/bit multi-channel reconfigurable GMSK/PSK/16-QAM transmitter with band-shaping. In Proceedings of the 2014 IEEE Asian Solid-State Circuits Conference (A-SSCC), Kaohsiung, Taiwan, 10-12 November 2014. 
27. Kim, S.R.; Ryu, H.G. Analysis and design of QAPM modulation based on multi-carrier using compressive sensing for low power communication. In Proceedings of the 2012 International Conference on ICT Convergence (ICTC), Jeju, Korea, 15-17 October 2012.

28. Cheffena, M. Performance evaluation of wireless body sensors in the presence of slow and fast fading effects. IEEE Sens. J. 2015, 15, 5518-5526. [CrossRef]

29. Wang, J.; Fujiwara, T.; Kato, T.; Anzai, D. Wearable ECG based on impulse radio type human body communication. IEEE Trans. Biomed. Eng. 2015. [CrossRef] [PubMed]

30. Liao, Y.; Leeson, M.S.; Higgins, M.D. An in-body communication link based on $400 \mathrm{MHz}$ MICS band wireless body area networks. In Proceedings of the 2015 IEEE 20th International Workshop on Computer Aided Modelling and Design of Communication Links and Networks (CAMAD), Guildford, UK, 7-9 September 2015.

31. Anzai, D.; Aoyama, S.; Yamanaka, M.; Wang, J. Impact of spatial diversity reception on SAR reduction in implant body area networks. IEICE Trans. Commun. 2012, 95, 3822-3829. [CrossRef]

32. Nagata, Y.; Hiraoka, M.; Akuta, K.; Abe, M.; Takahashi, M.; Jo, S.; Nishimura, Y.; Masunaga, S.; Fukuda, M.; Imura, H. Radiofrequency thermotherapy for malignant liver tumors. Cancer 1990, 65, 1730-1736. [CrossRef]

33. Kailas, A.; Ingram, M.A. Wireless aspects of telehealth. Wirel. Personal Commun. 2009, 51, 673-686. [CrossRef]

34. Yousaf, S.; Javaid, N.; Khan, Z.A.; Qasim, U.; Imran, M.; Iftikhar, M. Incremental relay based cooperative communication in wireless body area networks. Procedia Comput. Sci. 2015, 52, 552-559. [CrossRef]

35. Yigitel, M.A.; Incel, O.D.; Ersoy, C. QoS-aware mac protocols for wireless sensor networks: A survey. Comput. Netw. 2011, 55, 1982-2004. [CrossRef]

36. Javaid, N.; Ahmad, A.; Nadeem, Q.; Imran, M.; Haider, N. Im-Simple: Improved stable increased-throughput multi-hop link efficient routing protocol for wireless body area networks. Comput. Hum. Behav. 2015, 51, 1003-1011. [CrossRef]

37. Sandhu, M.; Javaid, N.; Jamil, M.; Khan, Z.; Imran, M.; Ilahi, M.; Khan, M. Modeling mobility and psychological stress based human postural changes in wireless body area networks. Comput. Hum. Behav. 2015, 51, 1042-1053. [CrossRef]

38. Hayajneh, T.; Almashaqbeh, G.; Ullah, S.; Vasilakos, A.V. A survey of wireless technologies coexistence in WBAN: Analysis and open research issues. Wirel. Netw. 2014, 20, 2165-2199. [CrossRef]

39. Kathuria, M.; Gambhir, S. Quality of service provisioning transport layer protocol for WBAN system. In Proceedings of the 2014 International Conference on Optimization, Reliability, and Information Technology (ICROIT), Haryana, India, 6-8 February 2014.

40. Otto, C.; Milenkovic, A.; Sanders, C.; Jovanov, E. System architecture of a wireless body area sensor network for ubiquitous health monitoring. J. Mob. Multimed. 2006, 1, 307-326.

41. Razzaque, M.A.; Javadi, S.S.; Coulibaly, Y.; Hira, M.T. QoS-aware error recovery in wireless body sensor networks using adaptive network coding. Sensors 2014, 15, 440-464. [CrossRef] [PubMed]

42. Monowar, M.M.; Hassan, M.M.; Bajaber, F.; Al-Hussein, M.; Alamri, A. Mcmac: Towards a MAC protocol with multi-constrained QoS provisioning for diverse traffic in wireless body area networks. Sensors 2012, 12, 15599-15627. [CrossRef] [PubMed]

43. IEEE/IOS Healthcare IT Standards. Available online: https://standards.ieee.org/findstds/standard/ healthcare_it.html (accessed on 30 April 2016).

44. European Union Framework Programme 7 'WBAN Architecture and Open Middleware'. Available online: http://daphne-fp7.eu/sites/default/files/D3.2\%20DAPHNE_WBAN_Open_Middleware.pdf (accessed on 30 April 2016).

45. Picazo-Sanchez, P.; Tapiador, J.E.; Peris-Lopez, P.; Suarez-Tangil, G. Secure publish-subscribe protocols for heterogeneous medical wireless body area networks. Sensors 2014, 14, 22619-22642. [CrossRef] [PubMed]

46. Rushanan, M.; Rubin, A.D.; Kune, D.F.; Swanson, C.M. Sok: Security and privacy in implantable medical devices and body area networks. In Proceedings of the 2014 IEEE Symposium on Security and Privacy (SP), San Jose, CA, USA, 18-21 May 2014.

47. Wu, W.H.; Bui, A.A.; Batalin, M.A.; Au, L.K.; Binney, J.D.; Kaiser, W.J. Medic: Medical embedded device for individualized care. Artif. Intell. Med. 2008, 42, 137-152. [CrossRef] [PubMed]

48. Animal Experimentation the Facts. Available online: http://www.bbc.co.uk/ethics/animals/using/facts.shtml (accessed on 30 April 2016). 
49. IEEE Standards Association 802.15.6-2012-Part 15.6: Wireless Body Area Networks. Available online: https://standards.ieee.org/findstds/standard/802.15.6-2012.html (accessed on 30 April 2016).

50. Reina, D.G.; Toral, S.L.; Barrero, F.; Bessis, N.; Asimakopoulou, E. The role of ad hoc networks in the internet of things: A case scenario for smart environments. In Internet of Things and Inter-Cooperative Computational Technologies for Collective Intelligence; Springer: Berlin, Germany, 2013; pp. 89-113.

51. Ghamari, M.; Janko, B.; Sherratt, R.S.; Harwin, W.; Piechockic, R.; Soltanpur, C. A survey on wireless body area networks for e-healthcare systems in residential environments. Sensors 2016, 16, 831. [CrossRef] [PubMed]

(C) 2016 by the authors; licensee MDPI, Basel, Switzerland. This article is an open access article distributed under the terms and conditions of the Creative Commons Attribution (CC-BY) license (http://creativecommons.org/licenses/by/4.0/). 